\title{
Shock wave structure in a lattice gas
}

\author{
James E. Broadwell ${ }^{\text {a) }}$ \\ Graduate Aeronautical Laboratories, California Institute of Technology, Pasadena, California 91125 \\ Donghee $\operatorname{Han}^{\text {b) }}$ \\ Stanford University, Stanford, California 445-706
}

(Received 14 September 2006; accepted 8 March 2007; published online 3 May 2007)

\begin{abstract}
The motion and structure of shock and expansion waves in a simple particle system, a lattice gas and cellular automaton, are determined in an exact computation. Shock wave solutions, also exact, of a continuum description, a model Boltzmann equation, are compared with the lattice results. The comparison demonstrates that, as proved by Caprino et al. ["A derivation of the Broadwell equation," Commun. Math. Phys. 135, 443 (1991)] only when the lattice processes are stochastic is the model Boltzmann description accurate. In the strongest shock wave, the velocity distribution function is the bimodal function proposed by Mott-Smith. (C) 2007 American Institute of Physics. [DOI: 10.1063/1.2723156]
\end{abstract}

\section{INTRODUCTION}

Discrete velocity and lattice gases have become tools for clarifying the Boltzmann equation ${ }^{1-3}$ and for fluid mechanics. ${ }^{4-6}$ The paper of Benzi et al. ${ }^{7}$ is an interesting general discussion of these ideas. In this paper the aim is to illustrate ideas and concepts in both areas. To this end, the lattice gas results are initially viewed as experimental and the model Boltzmann equation as the corresponding theory. For these purposes the two-dimensional lattice gas of Hardy and Pomeau ${ }^{8}$ is appropriate. It is sufficiently simple to allow a large number of particles to be followed exactly and yet has properties that lead to the formation of shock and expansion waves and to the formation of an equilibrium state. In addition the model Boltzmann equations for shock waves can be solved exactly.

\section{PROBLEM FORMULATION}

The Hardy-Pomeau model, a lattice version of a discrete velocity gas, ${ }^{9,10}$ consists of indistinguishable articles moving on a two-dimensional lattice with four fixed velocities. Particles move and collide according to the following rules. In each time step, all particles move to the adjacent site and then some collide. Collisions, which turn the collision partners through $90^{\circ}$, take place if and only if two particles with oppositely directed velocities occupy the same site and the collision destination velocity locations are empty. Mass and momentum are conserved in the moves and collisions. Particles pass through each other between sites. The system is reversible so that from any initial condition, an isolated system develops a long-term Poincaré cycle and returns to the initial state after sufficient time. This model has been used previously to demonstrate how thermodynamically irreversible processes can arise in microscopically reversible systems. ${ }^{11}$

It is emphasized that the model is not intended to represent a real gas. With only a single speed, the thermodynamics is not realistic. Further, the model solutions are not Galilean invariant. The value of the model is in the availability of exact model Boltzmann solutions for comparison with the lattice gas results.

\section{SHOCK AND EXPANSION WAVE GENERATION}

The model and the arrangement for generating the waves are as follows. Consider a square lattice with reflective boundaries at $y=0$ and $y=L$, and those at $x=0$ and $x=L$ at first periodic so that east and west moving particles leaving at one boundary enter at the other. In the following, particles moving north $(\mathrm{N})$, east $(\mathrm{E})$, south $(\mathrm{S})$, and west $(\mathrm{W})$ will sometimes be denoted by the numerals 1 to 4 . The gas has a mean velocity to the right when there are more E (2) particles than $\mathrm{W}$ (4) particles. This velocity $u$ is $c[n(2)$ $-n(4)] / n$, where $c$ is the particle velocity and $n(i)$ the particle number density, i.e., the number per site or area, and $n=\sum n(i)$. For zero $y$-velocity, $n(1)=n(3)$. After the gas equilibrates, the boundaries at $x=0$ and $L$ are made reflective (as if walls were inserted) and the gas comes to rest at each wall with a shock generated at the right boundary and an expansion wave at the left.

This arrangement for generating the shock waves is that used by Caflisch in his investigation of shock wave structure in a similar discrete velocity gas ${ }^{12}$ and by Nadiga et al. ${ }^{13}$ studying shocks in a nine-velocity cellular automaton. It may be noted that the shocks cannot be generated by piston motion. Particles reflected from a moving piston have the full range of velocities, a process not describable by discrete velocity models.

Density distributions $n(i)$ averaged over the $y$-columns and five adjacent times, and normalized by the free stream value, are shown in Figs. 1 and 2 for $u / c=0.5$ and 1, respectively. In the computations, the particle velocity, the lattice spacing, and the time step are unity.

\section{MODEL BOLTZMANN EQUATION}

The model equation for the distribution function is derived following the Boltzmann procedure. This function, 


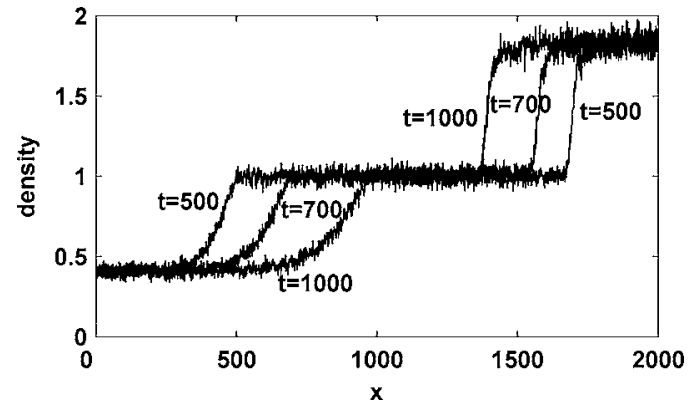

FIG. 1. Shock and expansion wave propagation, $u / c=0.50$.

$f(v(i), x, y, t)$, is the average number of particles with velocity $v(i)$ at location $(x, y)$, at time $t$, and is given by

$$
\frac{\partial f}{\partial t}+c_{x} \frac{\partial f}{\partial x}+c_{y} \frac{\partial f}{\partial y}=G-L,
$$

where $G$ and $L$ are the gain and loss from collisions, respectively.

For the lattice gas in which $n(i)$ is the particle number per unit area, this equation becomes the set of equations:

$$
\frac{\partial n(i)}{\partial t}+c_{x i} \frac{\partial n(i)}{\partial x}+c_{y i} \frac{\partial n(i)}{\partial y}=G(i)-L(i) .
$$

For north (1) moving particles loss occurs only when these particles collide with the south (3) particles. With the Boltzmann Stosszahlansatz - the assumption that there are no correlations in the particle motions - the collision rate depends only on the number densities of the colliding particles and their relative velocity. Thus, the loss $L_{1}$ is $2 \operatorname{con} n(1) n(3)$, where $2 c$ is the relative velocity and $\sigma$, in the usual derivation, is the collision cross section. Since particles move on grid lines and collide only when particles of oppositely directed velocity occupy the same site, $\sigma$ is taken to be the probability of this occurrence and, for dimensional consistency, $n(i)$ is viewed as the particles per unit horizontal length divided by the number of horizontal lines. In this way, $n(i)$ can continue to be viewed as the particle number per unit area. Particles are thrown into the velocity cell 1 by collisions between the 2 and 4 particles. Thus, with $\sigma$ taken to be $\frac{1}{2}$ (an assumption to be checked later), the equations become:

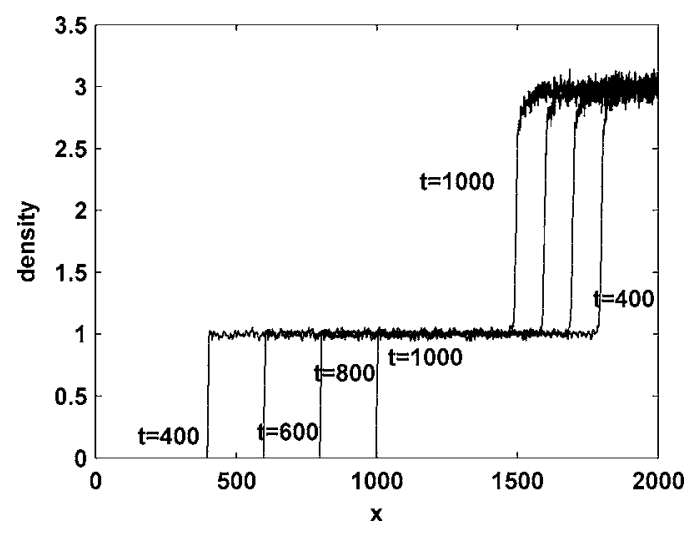

FIG. 2. Shock wave propagation, $u / c=1.0$.

$$
\begin{aligned}
& \frac{\partial n(1)}{\partial t}+c \frac{\partial n(1)}{\partial x}+c \frac{\partial n(1)}{\partial y} \\
& =G_{1}-L_{1}=c[n(2) n(4)-n(1) n(3)], \\
& \frac{\partial n(2)}{\partial t}+c \frac{\partial n(2)}{\partial x}+c \frac{\partial n(2)}{\partial y} \\
& =G_{2}-L_{2}=c[n(1) n(3)-n(2) n(4)], \\
& \frac{\partial n(3)}{\partial t}+c \frac{\partial n(3)}{\partial x}+c \frac{\partial n(3)}{\partial y} \\
& =G_{3}-L_{3}=c[n(2) n(4)-n(1) n(3)], \\
& \frac{\partial n(4)}{\partial t}+c \frac{\partial n(4)}{\partial x}+c \frac{\partial n(4)}{\partial y} \\
& =G_{4}-L_{4}=c[n(1) n(3)-n(2) n(4)] .
\end{aligned}
$$

These equations are put in nondimensional form by defining $n_{i}=n(i) / n_{a}$, where $n_{a}$ is the average field density; i.e., the total number of particles divided by the field area. For onedimensional flow in the $x$ direction, $n(1)=n(3)$, and the equations become:

$$
\begin{aligned}
& \frac{\partial n_{1}}{\partial \tau}=\left(n_{2} n_{4}-n_{1}^{2}\right), \\
& \frac{\partial n_{2}}{\partial \tau}+\frac{\partial n_{2}}{\partial \eta}=\left(n_{1}^{2}-n_{2} n_{4}\right), \\
& \frac{\partial n_{4}}{\partial \tau}-\frac{\partial n_{4}}{\partial \eta}=\left(n_{1}^{2}-n_{2} n_{4}\right),
\end{aligned}
$$

where $\tau=n_{a} c t, \eta=n_{a} x$.

This system of equations constitutes the Boltzmann description of the lattice gas. The populations $\left(n_{i}\right)$ make up the distribution function as they do for the discrete velocity gas.

\section{A. Shock wave propagation and structure}

In Fig. 1, where $u / c=0.50$ and $n_{a}=0.20$, the lattice is 2000 units long and 2000 units high. The number of particles at each site fluctuates between 0 and 4 . The shock propagates with a fixed shape while the expansion wave becomes less steep, as is the case in real gases. In Fig. 2 the initial condition is a stream of particles all with unit velocity to the right. In this case the gas separates from the left wall and a shock of density ratio three emerges at the right. The propagation speeds, structure, and distribution functions of the shock waves are discussed in the following and the results compared with the model Boltzmann equations approximations.

\section{B. Euler equation and shock jump conditions}

To study the shock wave structure, it is convenient to work in the coordinate system in which the shock is stationary. The shock speed and density jump are independent of the wave structure and can be determined from the inviscid Euler equation. This equation is obtained by sequentially multiplying Eqs. (1)-(3) by the collisional invariants, the 
molecular weight and particle velocities, and summing. The right-hand sides vanish because the particle number and momentum are conserved in the collisions. The results are:

$$
\begin{aligned}
& \frac{\partial \rho}{\partial \tau}+\frac{\partial \rho u}{\partial \eta}=0, \\
& \frac{\partial \rho u}{\partial \tau}+\frac{\partial\left(n_{2}+n_{4}\right)}{\partial \eta}=0,
\end{aligned}
$$

where

$$
\begin{aligned}
& \rho=m \sum n_{i}, \\
& u=\frac{m\left(n_{2}-n_{4}\right)}{\rho},
\end{aligned}
$$

and $m$ is the molecular weight (taken to be unity in the following).

The equations describe nonviscous adiabatic flows and thus flows in equilibrium; in the lattice gas this condition is $n_{2} n_{4}=n_{1}^{2}$.

With this condition, Eq. (5) can be written, following Caflisch,

$$
\frac{\partial \rho u}{\partial \tau}+\frac{\partial \rho F(u)}{\partial \eta}=0,
$$

where

$$
F(u)=(1 / 2)\left(1+u^{2}\right) .
$$

For the shocks in Figs. 1 and 2 moving to the left at velocity $s$ in a rightward flowing stream of velocity $u$, the jump conditions from the Euler equations are:

$$
\begin{aligned}
& \rho_{r}\left(u_{r}-s\right)=\rho_{l}\left(u_{l}-s\right), \\
& \rho_{r}\left(F_{r}-u_{r} s\right)=\rho_{l}\left(F_{l}-u_{l} s\right),
\end{aligned}
$$

where the subscripts $l$ and $r$ denote conditions upstream and downstream of the shock wave, respectively.

Noting that $F_{r} \equiv F\left(u_{r}\right)=1 / 2$, we find the shock speed in terms of $u$ to be

$$
s=\frac{1}{2}\left[u / 2 \pm \sqrt{\left(u^{2} / 4\right)+2}\right] .
$$

From the continuity equation (6), the density ratio is

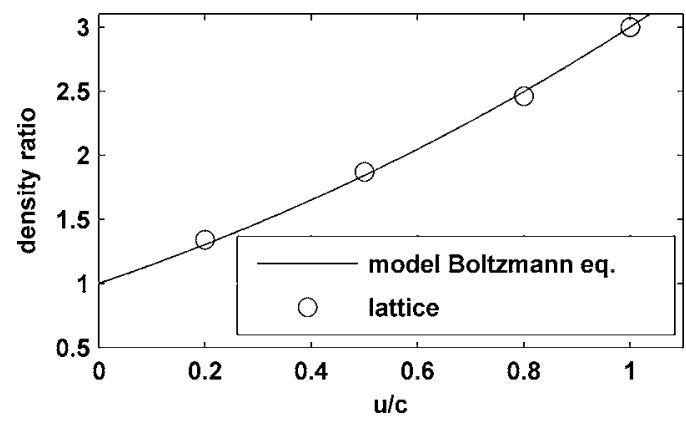

FIG. 3. Shock density ratio vs $u / c$.

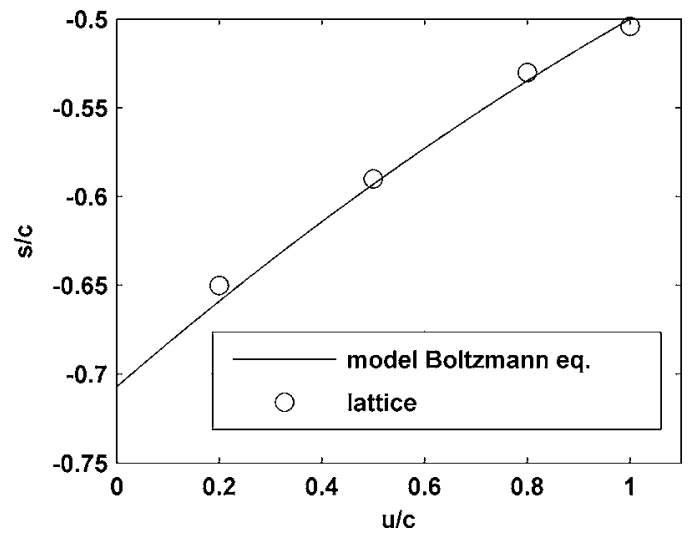

FIG. 4. Shock speed vs $u / c$.

$$
\frac{\rho_{r}}{\rho_{l}}=\frac{-(u-s)}{s} .
$$

From the results in Figs. 1 and 2, and for other speeds, Figs. 3 and 4 compare the density ratio and speed from these equations with those in the lattice. That the flow is in equilibrium entering and leaving the shock is shown later.

\section{Speed of sound}

Equations (6) and (7) indicate that as the density jump falls to zero, the wave speed is $1 / \sqrt{2}$. This is the speed of a wave in equilibrium. The structure of weak or acoustic waves not in equilibrium is described by linearized versions of Eqs. (1)-(3) for perturbations about the condition $u=0$, $n_{1}=n_{2}=n_{3}=n_{4}$. Denote the perturbations $n_{i}^{\prime}$, Equations (1)-(3) then lead to a single equation for $n_{i}^{\prime}$, for $n_{4}^{i}$, say:

$$
\frac{\partial}{\partial t}\left(\frac{\partial^{2} n_{4}^{\prime}}{\partial t^{2}}-c^{2} \frac{\partial^{2} n_{4}^{\prime}}{\partial x^{2}}\right)-4\left(\frac{\partial^{2} n_{4}^{\prime}}{\partial t^{2}}-\frac{c^{2}}{2} \frac{\partial^{2} n_{4}^{\prime}}{\partial x^{2}}\right)=0 .
$$

Moore and Gibson ${ }^{14}$ discuss equations of this form for gases out of vibrational or dissociation equilibrium, in which case $c$ is called the frozen speed and $c / \sqrt{2}$ the equilibrium speed. Whitham $^{15}$ showed that for equations of this type the wave propagating at speed $c$ decays exponentially and the low speed wave diffuses. (Similar results are demonstrated by Nadiga et $a .^{13}$ for the nine-velocity gas.) Here, particles leaving the wall at speed $c(=1$ in the model) begin, with every collision, to form the equilibrium state. Hence, the perturbation propagates only at $\mathrm{c} / \sqrt{2}$. Detailed results for a wave in a flow with $u / c=0.10$ are shown in Fig. 5. The relative fluctuations are large, but it is clear that no significant disturbance propagates at the speed $c$ and that the speed of the main disturbance is consistent with $c / \sqrt{2}$.

\section{Shock wave structure}

As was noted above, the model is not Galilean invariant; consequently, Eqs. (1)-(3) are different in this coordinate system. In a system moving with speed $s$, they are

$$
-s \frac{d n_{1}}{d \eta}=\left(n_{2} n_{4}-n_{1}^{2}\right)
$$




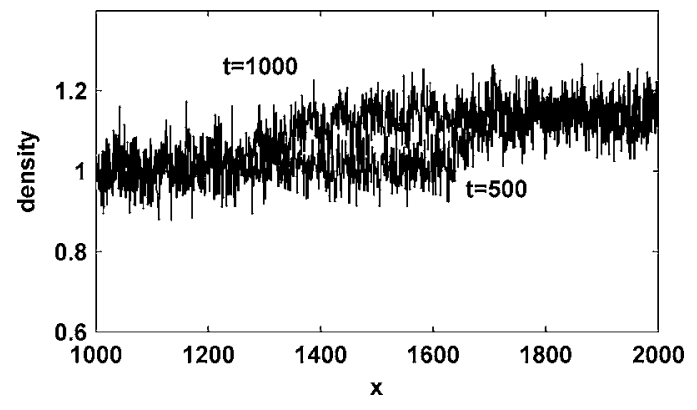

FIG. 5. Sound wave, $u / c=0.10$.

$$
\begin{aligned}
& (1-s) \frac{d n_{2}}{d \eta}=\left(n_{1}^{2}-n_{2} n_{4}\right), \\
& -(1+s) \frac{d n_{3}}{d \eta}=\left(n_{2} n_{4}-n_{1}^{2}\right) .
\end{aligned}
$$

Consider first $u_{1}=1$ in which all the free stream particles are moving east and $\left(n_{2}\right)_{i}=1$. From Eq. (6) $s=-1 / 2$. From these equations, $n_{1}$ and $n_{3}$ are functions of $n_{2}$, so that Eq. (9) can be written

$$
\frac{d n_{2}}{d \eta}=2\left(3-7 n_{2}+4 n_{2}^{2}\right)
$$

with the solution:

$$
n_{2}=\frac{\left(1-3 A e^{2 \eta}\right)}{\left(1-4 A e^{2 \eta}\right)},
$$

where

$$
A=\frac{\left[n_{2}(0)-1\right]}{\left[4 n_{2}(0)-3\right]},
$$

and

$$
n_{1}=n_{2}=3\left(1-n_{2}\right) .
$$

Note that $(n 2)_{i}$ identically unity produces the solution $n_{2}=1$; solutions applicable to lattice results are produced with $n_{2}(0)$ slightly less than 1 . Similar equations describe the motions for other free stream velocities.

Figures 6 and 7 compare the shock density profiles from the model Boltzmann equation solutions with the lattice profiles for $u / c=0.5$ and $u / c=1.0$. The axial locations of the shocks are arbitrary and the model and lattice profile locations are set equal. We see a considerable difference between

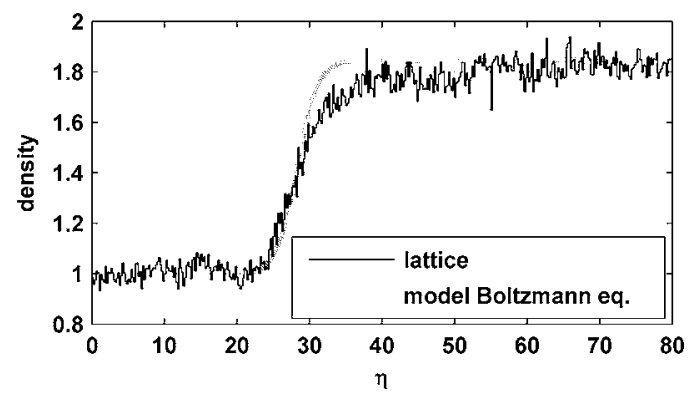

FIG. 6. Shock wave profile, deterministic gas, $u / c=0.50$.

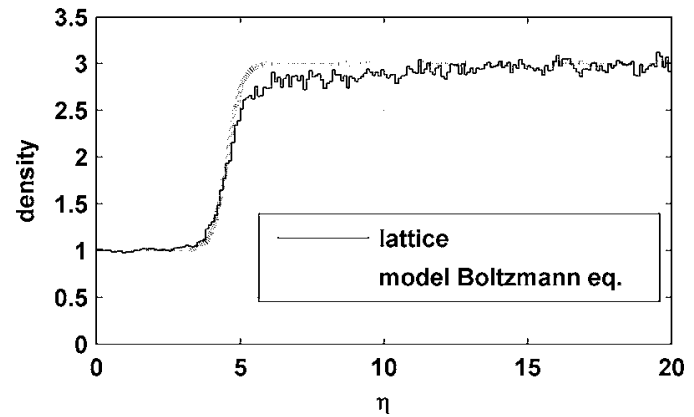

FIG. 7. Shock wave profile, deterministic gas, $u / c=1.0$.

the model solutions and the lattice gas shapes. (Changes in the collision cross section $\sigma$ do not affect the comparison.)

The analysis of Boghosian and Taylor ${ }^{16}$ concerning the development of correlations in lattice gases suggests that violations of the Boltzmann Stosszahlansatz are the source of the error. This observation together with the analysis of Caprino et al. ${ }^{17}$ showing for a similar model that "The stochasticity is absolutely necessary..." lead to the following investigation. The lattice program was modified to allow the collision probability to be specified. (With this step, the notion that the lattice results are experimental is dropped-in a real experiment, such a change is, of course, not possible.) This was done by assigning a random number between 0 and 1 to each site at every time step. For sites where the conditions for collision are met, collisions occur under the following additional condition. For a chosen probability-0.3, say-collisions are caused to occur at sites where the random number is less than 0.3 .

An effect of the reduced probability of collision is a reduced collision rate. In the model equation the effect is accounted for by making the effective density the product of the density and the probability $p n_{a}$. Under these conditions, Figs. 8 and 9 show that the model Boltzmann solutions closely describe the lattice profiles, results implying that correlations violating the Boltzmann Stosszahlansatz are the source of the errors in Figs 6 and 7.

The degree of departure from equilibrium is the magnitude of $k=n_{2} n_{4}-n_{1}^{2}$, the collision term in Eqs. (8)-(10). This function, together with the Boltzmann $H$-function, i.e., $H$ $=\sum n_{i} \ln n_{i}$, are shown in Fig. 10, where they are plotted on an

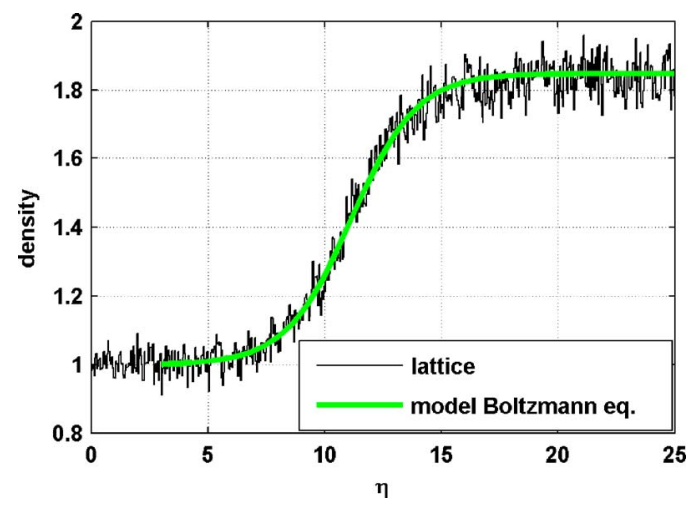

FIG. 8. Shock wave profile, stochastic gas, $u / c=0.50, n_{a}=0.20, p=0.50$. 


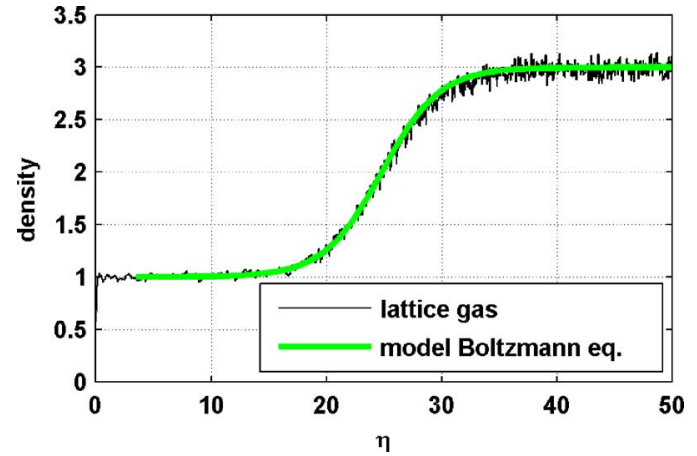

FIG. 9. Shock wave profile, stochastic gas, $u / c=1.0, n_{a}=0.20, p=0.05$.

arbitrary scale. There are small departures from equilibrium and fall in the $H$-function in the expansion wave and large changes in the shock.

\section{E. Velocity distribution function}

The velocity distribution function for the $u / c=1.0$ shock wave was discussed above and is given in Eqs. (12) and (13) as follows:

$$
n_{2}=\frac{\left(1-3 A e^{2 \eta}\right)}{\left(1-4 A e^{2 \eta}\right)},
$$

where

$$
A=\frac{\left[n_{2}(0)-1\right]}{\left[4 n_{2}(0)-3\right]} \text { and } n_{1}=n_{3}=3\left(1-n_{2}\right) .
$$

For the $u / c=0.5$ shock, this function was found by numerical integration of Eqs. (8)-(10). These solutions are compared with the stochastic lattice values in Figs. 11 and 12.

Mott-Smith was one of the first investigators to apply the Boltzmann equation in the study of shock wave structure. ${ }^{18}$ He postulated a binomial velocity distribution function in high Mach number waves that consists of a combination of the upstream and downstream Maxwellian functions. Narasimha and Das ${ }^{19}$ present an analytical solution of the Boltzmann equation for the infinitely strong shock that has precisely this form. That work suggested the following reexamination of the distribution function for the $u / c=1.0$ shock wave discussed above. That function can be put in the form proposed by Mott-Smith by subtracting $n_{1}$ from $n_{2}$, leaving $1 /\left(1-4 A e^{2 \eta}\right)$ to be denoted $n_{L}$, an incoming particle beam of declining strength. The subtracted term then be-

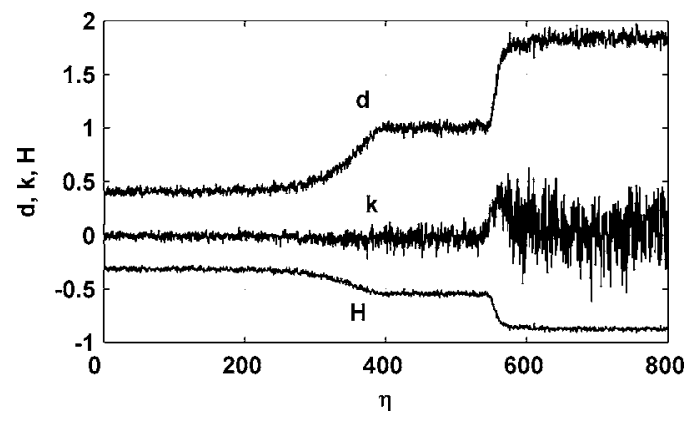

FIG. 10. Density ratio $k$ and $H$-function (arbitrary scale).

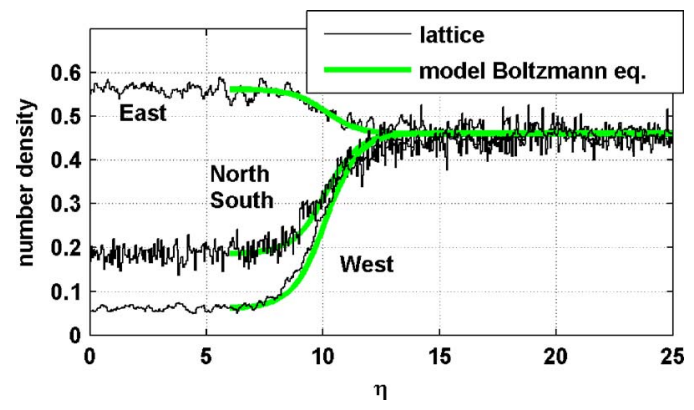

FIG. 11. Velocity distribution function, stochastic gas, $u / c=0.50$.

comes part of the rising strength exit equilibrium function $n_{R}$, consisting of $n_{1}+n_{2}+n_{3}+n_{4}$, all equal. These functions are compared with the lattice gas values in Fig. 13.

Narasima and Das describe their spectral method as reducing the Boltzmann equation to an equivalent infinite order nonlinear dynamical system. The functions in Fig. 13 are remarkably similar to those in their Fig. 5(a), the solution with a single mode. This solution describes the shock process as the scattering of an incoming particle beam into an exiting distribution function.

Such distributions are also observed in high Mach number shock waves. See Pham-Van-Diep et $a l^{20}$ and Alsmeyer ${ }^{21}$ for a further discussion.

\section{DISCUSSION AND CONCLUDING REMARKS}

The lattice results for the deterministic lattice gas have been considered experimental and compared to the model Boltzmann solutions as theory; the corresponding situation in real gases would be a comparison of Boltzmann equation solutions to experiments. In that case, of course, the collisions cannot be made probabilistic and general solutions to the Boltzmann equations are not available. However, the Bird direct simulation Monte Carlo (DSMC) method produces solutions to the Boltzmann equation. ${ }^{22,23}$ Thus, the remarkable agreement between the experiments and the DSMC solutions in, for instance, Pham-Van-Diep et al. ${ }^{20}$ and Alysmeyer, ${ }^{21}$ suggests that correlations do not develop in real gases.

Shock waves are simple examples of thermodynamically irreversible processes taking place in a microscopically reversible system. Confusion in this subject comes from a failure to distinguish between macroscopic and microscopic ir-

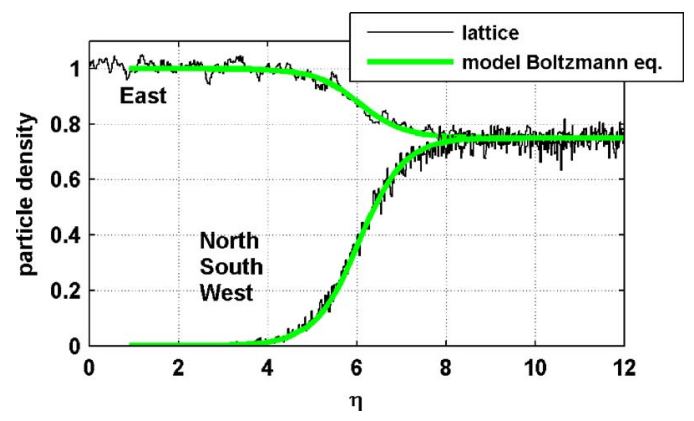

FIG. 12. Velocity distribution function, stochastic gas, $u / c=1.0$. 


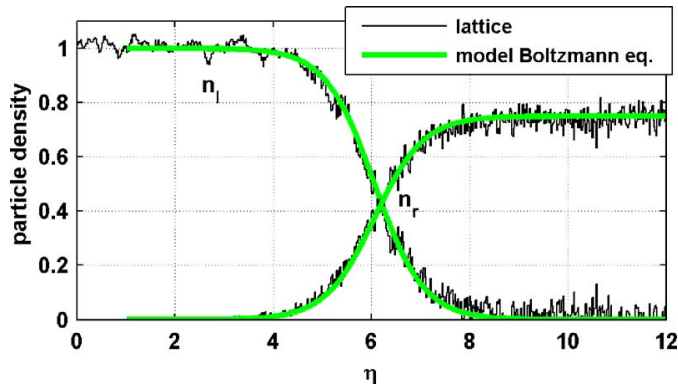

FIG. 13. Mott-Smith distribution function, stochastic gas, $u / c=1.0$.

reversibility. In this model the difference is clear: the particle collisions and motions are reversible and the change in the $H$-function across the shock is a property of the collection of particles.

Finally, the departure from equilibrium and fall in the $H$-function in the expansion wave are reminders that in fluid mechanics such waves are treated as isentropic, usually without any remark about the approximation.

\section{ACKNOWLEDGMENTS}

We thank Russel Caflisch for his participation in the initial modeling work and Roddam Narasimha, Anatol Roshko, and John Torczynski for their helpful comments. We especially thank Godfrey Mungal for his suggestions concerning many aspects of the work.

${ }^{1}$ A. Palczewski, J. Schneider, and A. Bobylev, "A consistency result for a discrete-velocity model of the Boltzmann equation," SIAM (Soc. Ind. Appl. Math.) J. Numer. Anal. 34, 1865 (1997).

${ }^{2}$ H. J. Higuera and J. Himenez, "Boltzmann approach to lattice gas simulations," Europhys. Lett. 7, 663 (1989).

${ }^{3}$ H. Chen, S. Kandasamy, S. Orszag, R. Shock, S. Succi, and V. Yakhot, "Extended Boltzmann kinetic equation for turbulent flows," Science 301, 663 (2003).

${ }^{4}$ S. Chen and G. D. Doolen, "Lattice Boltzmann method for fluid flows," Annu. Rev. Fluid Mech. 30, 329 (1998).
${ }^{5}$ F. J. Higuera and S. Succi, "Simulating the flow around a circular cylinder with a lattice Boltzmann equation,” Europhys. Lett. 8, 517 (1989).

${ }^{6}$ F. J. Higuera, S. Succi, and R. Bemzi, "Lattice gas dynamics with enhanced collisions," Europhys. Lett. 9, 345 (1989).

${ }^{7}$ S. Benzi, S. Succi, and M. Vergassola, "The lattice Boltzmann equation; theory and applications," Phys. Rep. 222, 145, (1992).

${ }^{8}$ J. Hardy and Y. Pomeau, "Thermodynamics and hydrodynamics for a modeled fluid," J. Math. Phys. 13, 1042 (1972).

${ }^{9}$ J. E. Broadwell, "Study of rarefied shear flow by the discrete velocity method," J. Fluid Mech. 19, 401 (1964).

${ }^{10}$ J. E. Broadwell, "Shock structure in a simple discrete velocity gas," Phys. Fluids 7, 1243 (1964).

${ }^{11}$ J. E. Broadwell, "Irreversibility in a reversible lattice gas," J. Stat. Phys. 1035-1036, 1125 (2001).

${ }^{12}$ R. E. Caflisch, "Navier-Stokes and Boltzmann shock profiles for a model of gas dynamics," Commun. Pure Appl. Math. 32, 521 (1979).

${ }^{13}$ R. T. Nadiga, J. E. Broadwell, and B. Sturtevant, "Study of a multispeed cellular automaton," in Rarefied Gas Dynamics: Theory and Computational Techniques: 16th International Symposium, Pasadena, CA, July 1016, 1988 [Progress in Astronautics and Aeronautics, edited by E. P. Muntz, D. P. Weaver, and D. H. Campbell (AIAA, Washington, DC, 1989), Vol. 118, pp. 155-170].

${ }^{14}$ F. K. Moore and W. E. Gibson, "Propagation of a weak disturbance in a gas subject to relaxation effects," J. Aerosp. Sci. 27, 117 (1960).

${ }^{15} \mathrm{G}$. B. Whitham, "Some comments on wave propagation and shock wave structure with applications to magnetohydrodynamics," Commun. Pure Appl. Math. 12, 113 (1959).

${ }^{16} \mathrm{~B}$. M. Boghosian and W. Taylor, "Correlations and renormalizations in lattice gases," Phys. Rev. E 52, 510 (1995).

${ }^{17}$ S. Caprino, A. DeMasi, E. Presutti, and M. Pulvirenti, "A derivation of the Broadwell equation," Commun. Math. Phys. 135, 443 (1991).

${ }^{18}$ H. M. Mott-Smith, "The Solution of the Boltzmann equation for a shock wave," Phys. Rev. 82, 885, (1951).

${ }^{19}$ R. Narasimha and P. Das, "A spectral solution of the Boltzmann equation for the infinitely strong shock," Philos. Trans. R. Soc. London, Ser. A 330, 217 (1990).

${ }^{20}$ G. Pham-Van-Diep, D. Erwin, and E. P. Muntz, "Nonequilibrium molecular motion in a hypersonic shock wave," Science 245, 624 (1989).

${ }^{21} \mathrm{~S}$. Alsmeyer, "Density profiles in argon and nitrogen shock waves measured by the absorption of an electron beam," J. Fluid Mech. 74, 497 (1976).

${ }^{22}$ G. A. Bird, "Direct simulation and the Boltzmann equation," Phys. Fluids 13, 2676, (1970).

${ }^{23} \mathrm{~W}$. Wagner, "A convergence proof for the Bird's direct simulation Monte Carlo method for the Boltzmann equation,” J. Stat. Phys. 66, 1011 (1992). 